\title{
In Situ High Temperature Atomic Level Studies of Large Closed Grain Boundary Loops in Graphene
}

\author{
Chuncheng Gong ${ }^{1}$, Kuang He ${ }^{1}$, Qu Chen ${ }^{1}$, Alex W. Robertson ${ }^{1}$, Jamie H. Warner ${ }^{1 *}$ \\ ${ }^{1}$ Department of Materials, University of Oxford, Parks Road, Oxford, OX1 3PH, United Kingdom \\ Email: *Jamie.warner@materials.ox.ac.uk;
}

\begin{abstract}
We use an in situ heating holder within an aberration corrected transmission electron microscope (AC-TEM) to study the structure and dynamics of large closed grain boundary (GB) loops in graphene at the atomic level. Temperatures up to $800^{\circ} \mathrm{C}$ are used to accelerate dynamic evolution of the defect clusters, increasing bond rotation and atomic addition/loss. Our results show that the large closed GB loops relax under electron beam irradiation into several isolated dislocations far apart from each other. Line defects composed of several adjacent adatom clusters can be found during the reconfiguration process. Dislocation ejection from the closed GB loops are seen in real time and are shown to help the reduction in loop size. These results show detailed information about the stability and behavior of large GB loops in 2D materials that have importance in the high temperature processing of these materials.
\end{abstract}

KEYWORDS: Graphene, dislocations, grain boundary, TEM 
Defects in graphene have a great impact on its exceptional electronic, mechanical and chemical properties. ${ }^{1-5}$ Compared with other defects, grain boundaries (GBs) have attracted much more attention recently since their presence is inevitable in polycrystalline graphene synthesized by chemical vapor deposition (CVD). The atomic structure of GBs in graphene has been revealed by multiple TEM and STM studies, which is, in most cases, a chain of adjacent pentagon-heptagon dislocation cores. ${ }^{6-10}$ Lahiri et al. has also found 5-8-5 configured linear GB in graphene, the formation of which is due to the strong interaction with Ni (111) substrate. ${ }^{11}$ Most often GBs in graphene are classified as symmetric and asymmetric. GBs in CVD synthesized graphene are often highly asymmetric and meandering, which could reconstruct into periodic and symmetric forms after thermal annealing or electron irradiation. ${ }^{12,13}$ Theoretic calculations have shown that the symmetric GB in graphene increases its mechanical strength and introduces magnetism. ${ }^{14-16}$ Yazyev et al. have reported the presence of a well-defined transport gap across a particular asymmetric graphene GB. ${ }^{17}$ These studies suggest that the GB is a promising candidate for graphene defect nanoengineering and nanoscale device fabrication.

However, there is also a third type of GB where the pentagon-heptagon pairs are end-toend connected, generating a loop with inner honeycomb lattice rotated, known as the closed GB loop. To date very few research efforts have been devoted to investigating such defect in either theory or experiment. Kurasch et al. have observed GB loops with 1-2 nm diameters and have shown that their structural change is mediated by bond rotations with both real-time TEM observation and Monte Carlo simulation. ${ }^{9}$ The presence of such defect in graphene is also predicted to decrease the thermal conductivity of the material. ${ }^{18}$ However, the formation dynamics of the GB loop still remains mysterious. Its stability and detailed structural evolution also need to be confirmed by further experiment. 
In this work we examine the atom-by-atom transformations associated with large closed loop GB defects using high temperature in situ AC-TEM. Monolayer graphene was synthesized by ambient pressure CVD method on a melted copper substrate as previously reported. ${ }^{19}$ The sample was then transferred onto a SiN designed for in situ temperature controlled TEM experiments up to $900^{\circ} \mathrm{C}$. AC-TEM imaging was performed at an accelerating voltage of $80 \mathrm{kV}$ utilizing an image corrector, which allows Angstrom resolution imaging of the topological defects in graphene.

\section{Results and Discussion}
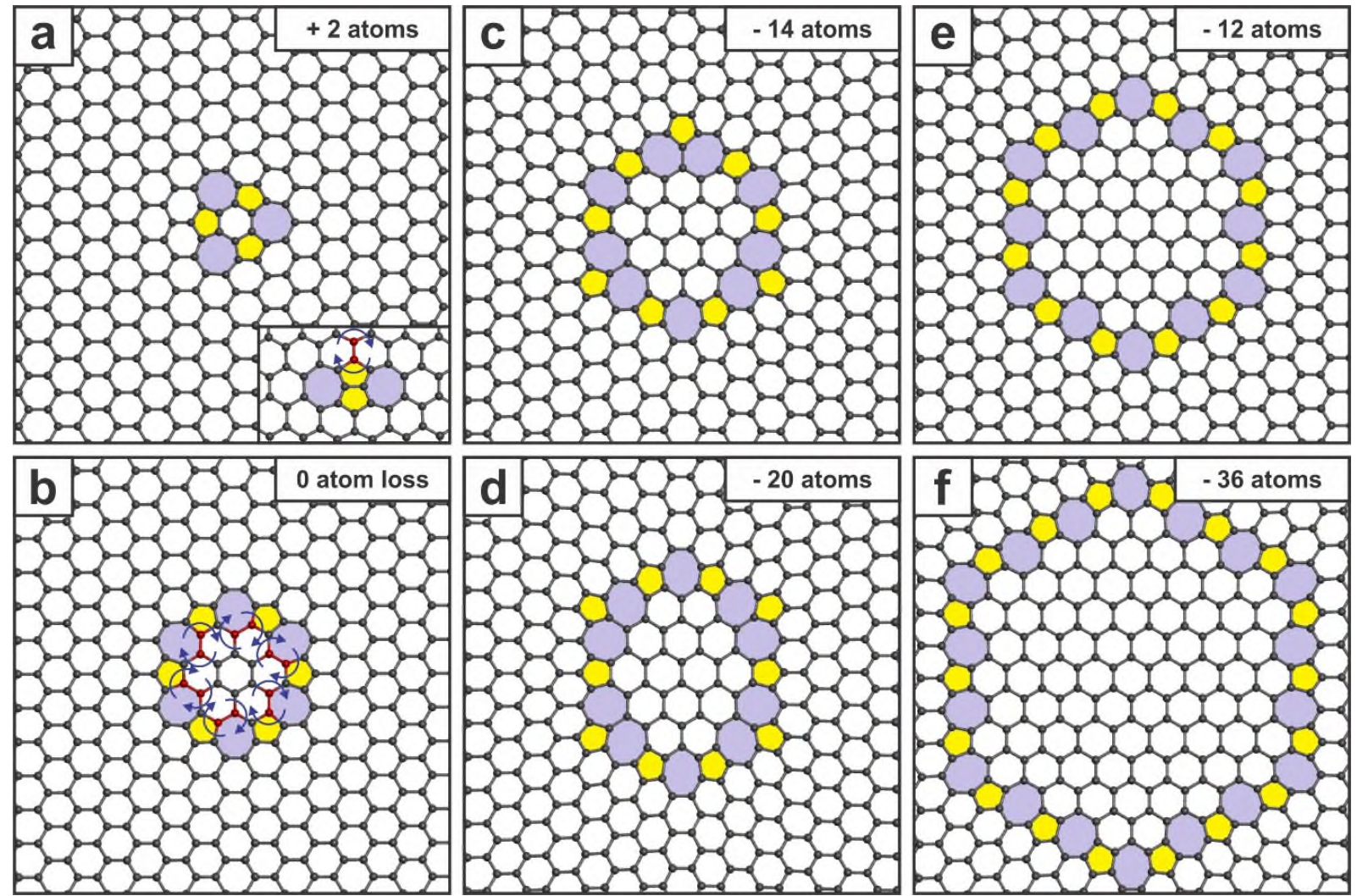

Figure 1. Atomic models of multiple closed GB loop defects with different sizes and symmetries. (a) A blister defect with 2 additional carbon atoms incorporated into the graphene lattice. (b) The most commonly observed closed GB defect containing a seven-hexagon core rotated by $30^{\circ}$. (c) Three-fold symmetric closed GB loop defect with 14 atoms lost. (d) Two-fold symmetric closed GB loop with 20 atoms removed from the lattice. (e, f) Two six-fold symmetric closed GB with 10 and 36 carbon atoms lost, respectively. 
Figure 1 illustrates several different closed GB loop defects constructed of adjacent 5-7 dislocations, with the number of additional or subtractive atoms required in their formation processes. Figure 1a is the smallest carbon adatom defect with two additional atoms incorporated into the graphene lattice. The three-fold symmetric defect contains one hexagon in the core rotated by $30^{\circ}$ with respect to the bulk lattice, surrounded by a triad of 5-7 edge dislocations. ${ }^{20}$ This defect can be transformed from an inverse Stone-Wales defect via a single bond rotation, as shown in the inset in Figure 1a. ${ }^{21}$ Previous research has shown that adatom defects are energetically unfavorable under electron irradiation and the additional atoms easily get sputtered. ${ }^{20}$ The flower-like defect containing a seven-hexagon core in Figure $1 \mathrm{~b}$ has been reported in a large number of HRTEM studies, which can be relaxed by $6 \mathrm{SW}$ bond rotations..$^{9,13,22}$ It has been proposed to have relatively good stability with a low formation energy of $7.0 \mathrm{eV}$ (obtained from DFT calculations) while the activation energy for a $\mathrm{SW}$ rotation in pristine graphene is $9-10 \mathrm{eV} \cdot{ }^{23,24}$ However, it becomes difficult for the graphene lattice to have zero atomic loss when creating GB loops with larger sizes. In Figure 1c-e we identify 3-fold, 2-fold and 6-fold symmetric GB loops containing 12, 14 and 31 rotated hexagons respectively. The formation of each defect involves the removal 10-20 carbon atoms from pristine graphene lattice. In addition, with the increase in the loop perimeter more subtractive atoms are also required, as shown in Figure 1e and f. 

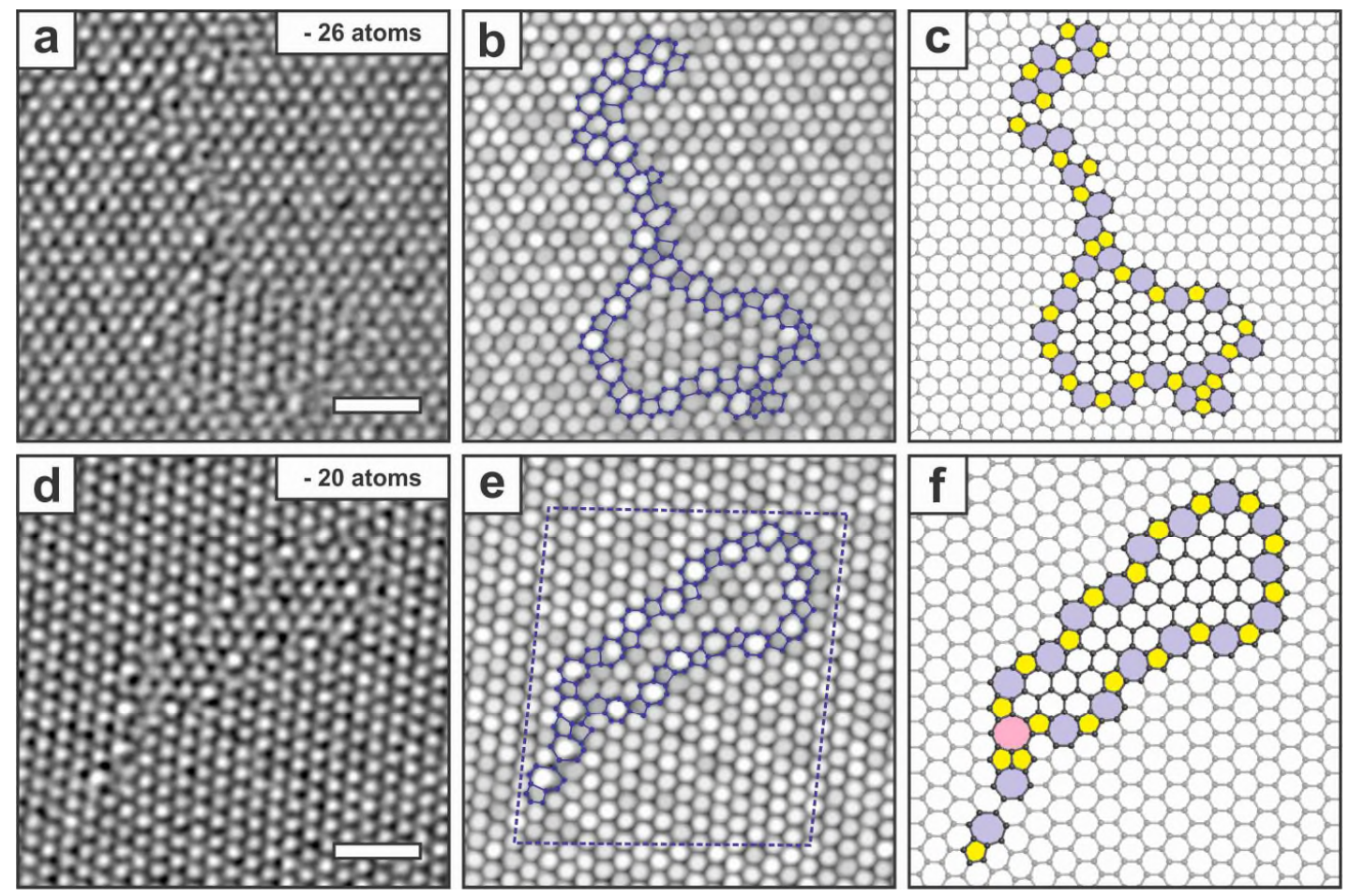

Figure 2. Two closed GB loops observed in monolayer graphene. (a, d) Gaussian filtered AC-TEM images of two closed grain boundary loops, the formation of which requires the removal of 26 and 20 atoms respectively. (b, e) Maximum filtered images calculated from $(a, d)$ with non-hexagonal rings highlighted in blue. The Burger's circuit around the defect in panel e is marked by a dashed parallelogram. (c, f) Accompanying atomic models of (a, d) with the color scheme indicating the number of carbon atoms in each ring: $5=$ yellow, $7=$ blue and $8=$ pink. Scale bars in panel a and $\mathrm{d}$ are $1 \mathrm{~nm}$.

Experimentally, large closed GB loops were occasionally found within the graphene lattice after heating to high temperature. We also used focused electron beam irradiation of pristine graphene areas at elevated temperatures of $500^{\circ} \mathrm{C}$ to also artificially create large closed GB loops in graphene to study. This is based on similar methods to create defects in graphene at room temperature and partial dislocations at high temperatures of $800^{\circ} \mathrm{C} . .^{22,25}$ AC-TEM images of two different closed GB loop structures are shown in Figure 2. The GB loop in Figure 2a contains 34 rotated hexagons within the defect, which is significantly larger than prior reports. Compared with the models we 
present in Figure 1, the experimentally observed GB loops in Figure 2 are less symmetric and regular in shape, and are often accompanied with other point defects. In rare cases, octagonal rings and distorted hexagonal rings are also found within the boundary loop. In Figure $2 \mathrm{~b}$ and e, a closed circuit is drawn around the defect to measure their Burger's vectors, which represent the magnitude and direction of dislocation-induced lattice deformation. We find that the Burger's vector for both GB loops is 0 , indicating there is no unpaired dislocation inside the defect. As a result, we are then able to determine that 26 and 20 atoms are sputtered in the formation processes of the GB loops in Figure 2. The detailed mechanisms of how this kind of defect is produced are not yet fully uncovered by experimental observations.

The GB loop in Figure 2a is found in graphene at $500{ }^{\circ} \mathrm{C}$ without any focused electron beam irradiation and is likely to be an intrinsic defect coming from the CVD growth process or from the high temperature heating process. At room temperature, the defects act as anchors for the surface carbon contamination and this prevents their identification by AC-TEM. However, heating the sample to elevated temperatures above $500^{\circ} \mathrm{C}$, typically mobilizes the contamination and eventually causes it to evaporate off the surface of graphene and leave the clean lattice structure beneath for AC-TEM imaging. The defect in Figure $2 b$ is created by exposing the pristine graphene to the focused electron beam for $1 \mathrm{~min}$ at $500{ }^{\circ} \mathrm{C}$. At room temperature, defect structures with more than 20 atoms loss resulted from prolonged focused beam exposure typically lead to nano-sized pores. The carbon atoms at the edge are under-coordinated and have a tendency to reconstruct into saturated vacancy structures. Previous research has suggested that the displacement cross section for carbon atoms in graphene increases with temperature. ${ }^{12}$ It is particularly apparent in the defective area when temperature is above $500{ }^{\circ} \mathrm{C}$. Therefore, a higher frequency of structural changes driven by bond rotations or atom evaporation is expected at elevated temperatures $(\geq 500$ 
$\left.{ }^{\circ} \mathrm{C}\right)$. Figure 3 reveals a possibility of how large GB loops can be formed using a focused electron beam at elevated temperatures. The beam is first focused within a $10-\mathrm{nm}$ diameter spot to significantly increase the beam current density. The exposure time can be varied for the purpose to create different types of defects. After that the beam is expanded back to image the graphene lattice. Figure 3a shows an example where a sub-nanopore and a multivacancy were initially generated by the sputtering damage at $800{ }^{\circ} \mathrm{C}$. The increased thermal energy together with beam irradiation enable rapid reconfiguration of the defect into a structure with lower formation energy. As shown in Figure 3, the multivacancy connected to the sub-nanopore after 4 min $35 \mathrm{~s}$ (Figure $3 b)$ and then filled the pore after another 6 mins, yielding a GB loop defect with almost all carbon atoms saturated (Figure 3c). This helps explains why the closed GB loop with size shown in Figure 2 is more likely to be observed at temperatures above $500{ }^{\circ} \mathrm{C}$, as the defects at room temperature lack the sustainable energy supply to restructure.
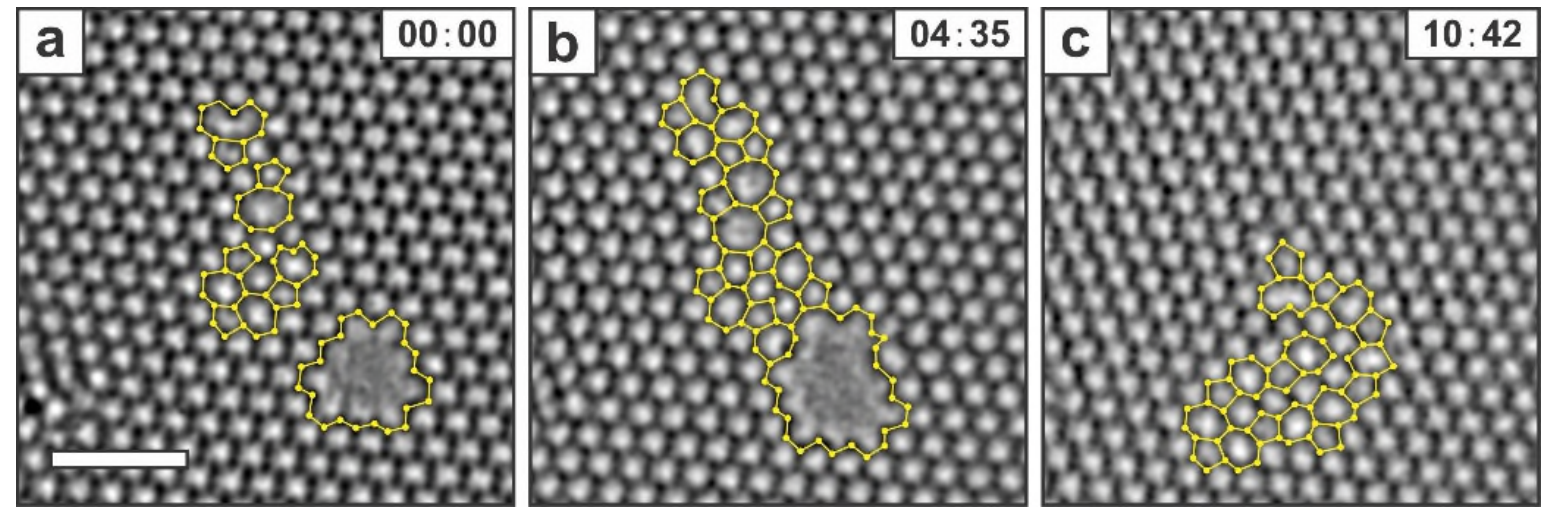

Figure 3. Time series of AC-TEM images showing the coalescence of a multivacancy and a sub-nanometer pore in monolayer graphene. (a) A multivacancy and a sub-nanometer pore are created by sputtering damage under electron irradiation. (b) Two defects get attached. (c) The vacancy and the pore get further merged, yielding a GB loop containing 6 hexagons. Non-hexagonal rings and dangling bonds are marked by yellow balls and sticks in all panels. The scale bar in panel a is $1 \mathrm{~nm}$. 
The image sequence in Figure 4 shows the structural evolution of the GB loop, initially shown in Figure $2 \mathrm{~d}$, from creation to annihilation. The rate of structural change is significantly accelerated at $500{ }^{\circ} \mathrm{C}$, which facilitates our investigation. Our data set in total consists of 130 frames recorded over $28 \min 8 \mathrm{~s}$. The acquisition interval between two consecutive frames is $\sim 10-15 \mathrm{~s}$. The GB loop was changing frequently throughout the transformation process. Structural reconfiguration was typically observed between two consecutive images, as seen from the time line chart of loop size in Figure 4q. The GB loop was relatively stable between $2 \min 4 \mathrm{~s}$ and $7 \mathrm{~min} 42 \mathrm{~s}$, as indicated by the larger blue ellipse in Figure 4q. This is probably due to the presence of the partial-dislocation like "tail" (Figure 2b). Previous research has shown that partial-dislocations incur less out-ofplane distortions than perfect dislocations. ${ }^{25}$ Thus, the "tail" in Figure $2 \mathrm{~b}$ may help to accommodate the rippling introduced by the GB loop and reduce the displacement threshold for atoms within the defect. At around $8 \mathrm{~min}$, the defect split into two separate loops as shown in Figure $4 c$. Images showing their subsequent structural evolution are presented in Figure $4 \mathrm{~d}-\mathrm{j}$ and Figure $4 k-p$ respectively. The loop at the bottom left in Figure $4 c$ first evolved into an extend line defect made up of a string of non-hexagonal rings, as shown in Figure 4k. The line defect then got gradually unwound in the next $8 \mathrm{~min}$ and reconstructed into a 5-7 dislocation at $18 \mathrm{~min} 4 \mathrm{~s}$, which then rapidly moved out of the imaging area within $12 \mathrm{~s}$ (Figure $4 \mathrm{p}$ ). The loop at the top right of Figure $3 \mathrm{c}$, however, maintained the closed loop configuration for quite some time. It first continued to grow larger and attained the maximum size at around $19 \mathrm{~min}$. Thereafter, the defect started to shrink until it reached a "double-flower" configuration as shown in Figure 4h. The double flower defect is the assembly of two flower defects illustrated by the model in Figure 1b which was robust under beam irradiation. After being stable for $2 \min \pm 13 \mathrm{~s}$, it was observed to unwind into a single 
flower defect via 6 bond rotations (Figure 4i). Soon afterwards the single flower defect also quenches via bond rotations.

The result in Figure 4 may give the impression that the closed GB loop defect is fully relaxed to pristine lattice after a nearly $30 \mathrm{~min}$ of continuous beam exposure at $500{ }^{\circ} \mathrm{C}$. However, examination a wider region of the area after the final frame showed that two dislocation cores far apart from each other were left (Figure 5a). Taking into account the dislocations in the bottom left of Figure 4o (position indicated by Figure $5 \mathrm{~b}$ inset), the five dislocations can be regarded as two pairs with their slip planes indicated by the blue and orange lines in Figure 5b. By counting the number of slip planes between the parallel lines, we could tell that 110 carbon atoms have been removed to generate these two dislocation pairs. Since there are possibly other undetected dislocations (such as the one paired with the 5-7 dislocation in the upper inset in Figure 5b) and the dislocations in the insets could make further climb motions after migrating out of the imaging region, 110 is the minimum number of missing atoms. Therefore, the majority of atom losses occurs during the shape evolution process. This is also confirmed in the frame-by-frame structural study in Figure 6, where the structural reconfiguration of the GB loop is not only driven by bond rotations as previously reported (Figure $6 \mathrm{a}, \mathrm{b}) .{ }^{9}$ Occasional carbon dimer evaporation within the defect is also inevitable under $80 \mathrm{kV}$ electron irradiation (Figure 6c, d). Previous work has demonstrated that dislocation in graphene is a very stable form of defect. ${ }^{\mathbf{2 6}-28}$ It can only annihilate by combining with an opposite dislocation or migrate to the edge of graphene. ${ }^{29}$ Thus, it can be concluded that isolated dislocations are the terminal state of a GB loop. 


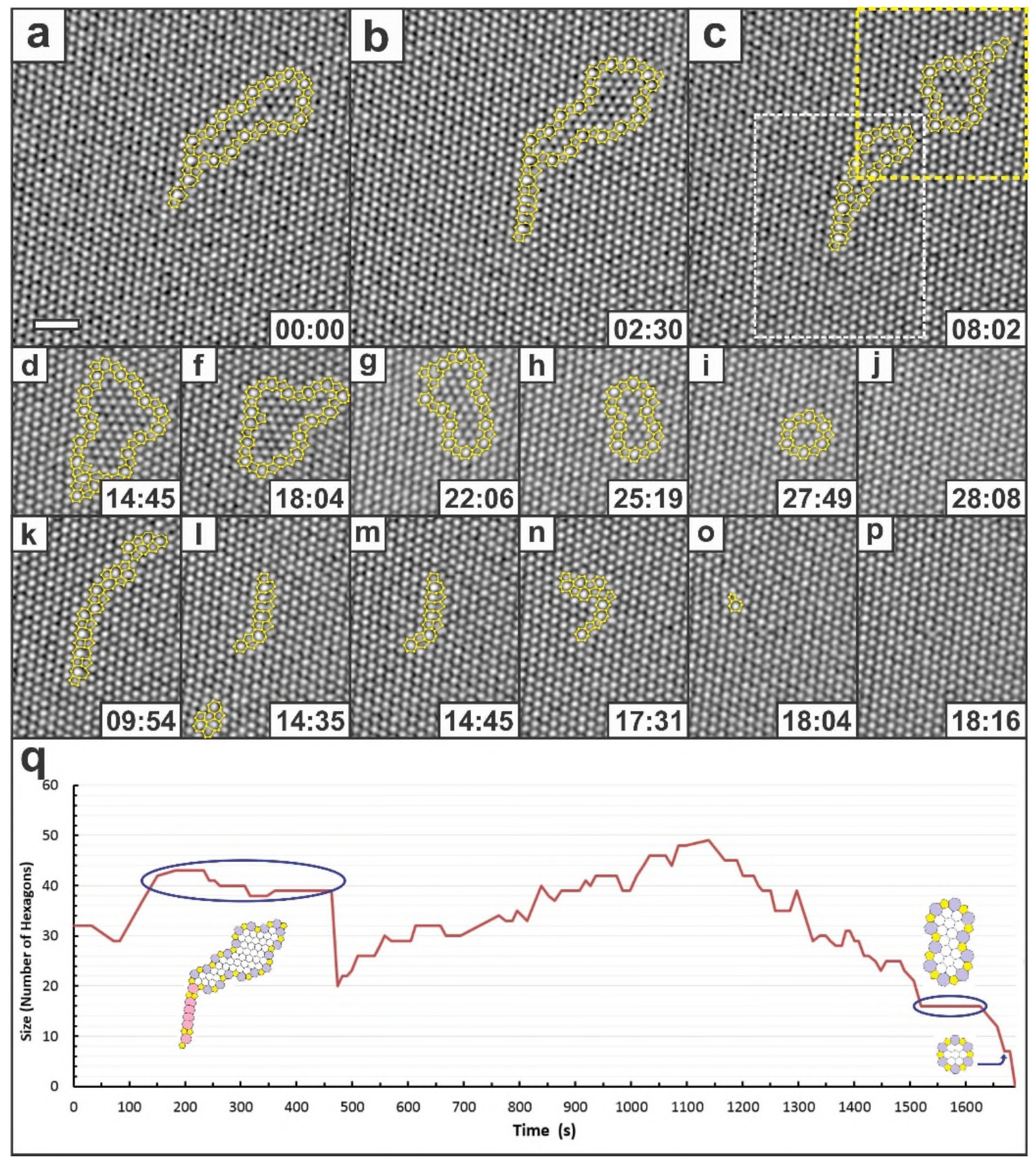

Figure 4. Structural evolution of the closed GB loop shown in Figure $2 \mathrm{~d}$ at $500{ }^{\circ} \mathrm{C}$. (a-c) Three time series frames showing the structural change of a GB loop in 8 min $2 \mathrm{~s}$. In panel c, the initial GB loop splits into two smaller ones. The unwinding processes for both of them are presented in $(d-j)$ and $(k-p)$. (q) Line chart indicating the size change of the GB loop with time by counting the number of undistorted hexagons within the defect. The scale bar in panel a is $1 \mathrm{~nm}$. 

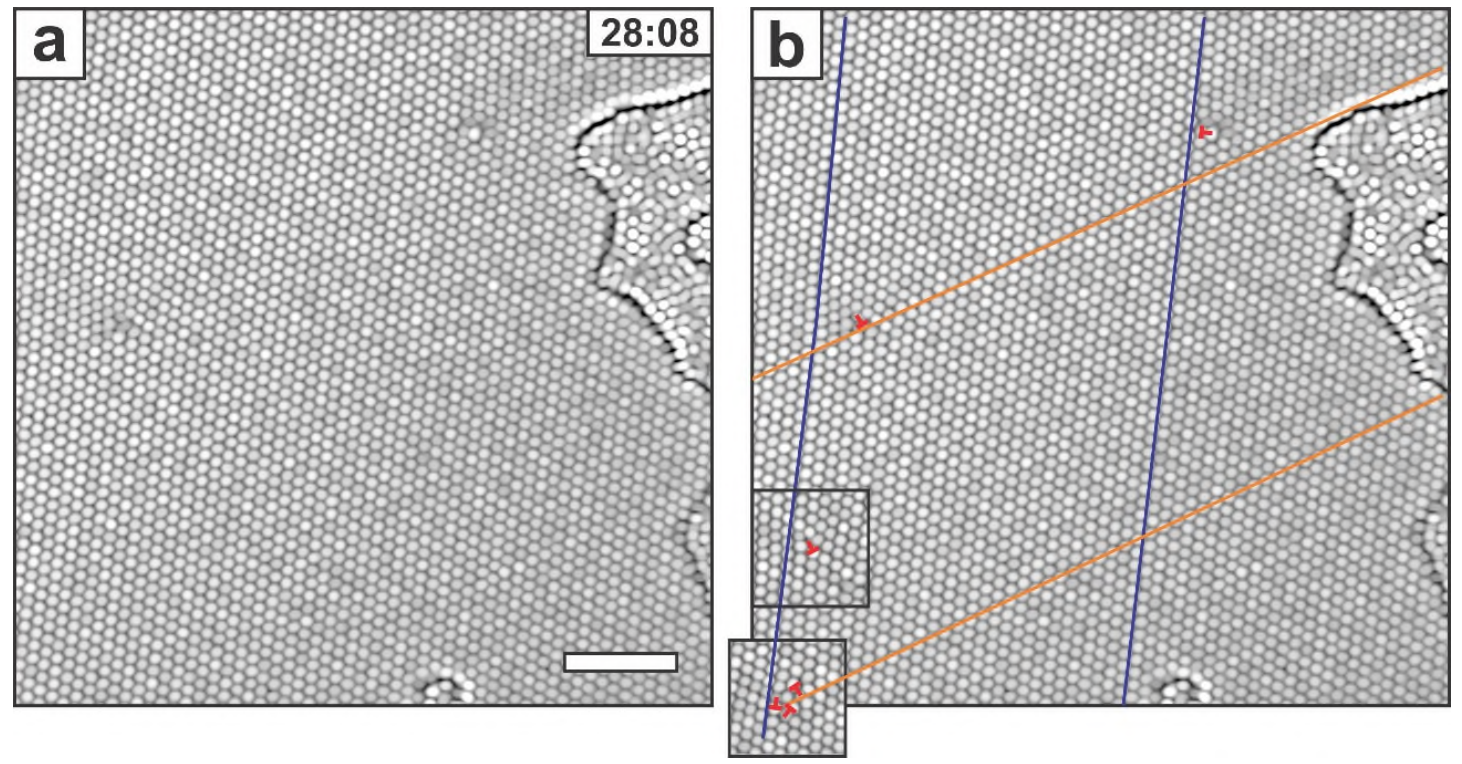

Figure 5. Maximum filtered AC-TEM image showing the final state of the GB loop shown in Figure 4 after 28 min $8 \mathrm{~s}$ beam irradiation. (a) Maximum filtered AC-TEM image. (b) Same TEM image as (a) with dislocations and their slip planes highlighted and overlapping insets showing the image of the same area at $18 \mathrm{~min} 4 \mathrm{~s}$ (upper) and 14 $\min 35 \mathrm{~s}$ (bottom), respectively. The scale bar in panel a is $2 \mathrm{~nm}$. 

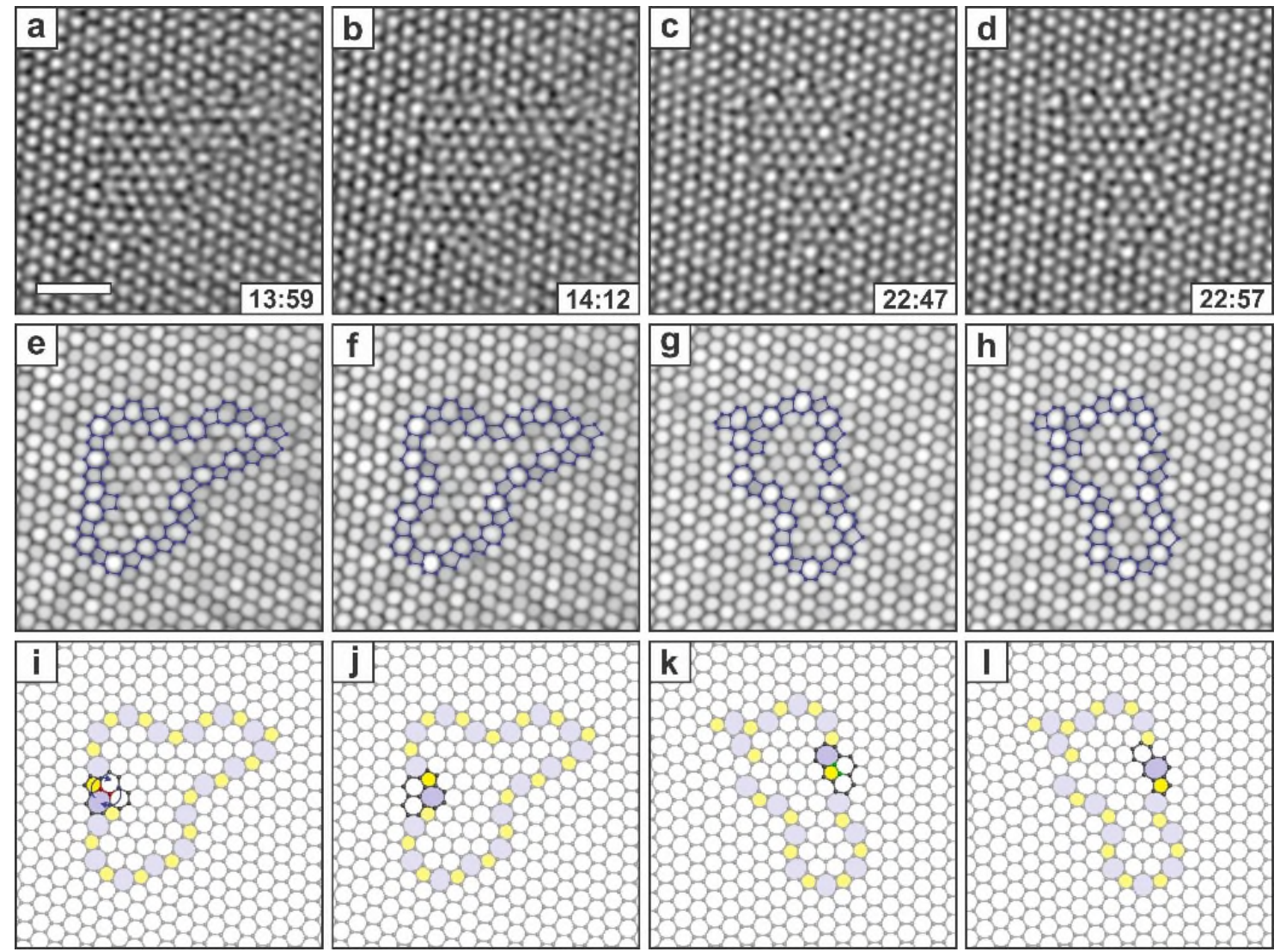

Figure 6. Structural change of the GB loop driven by bond rotation and carbon evaporation. (a, b) Bond rotation. (c, d) Carbon dimer evaporation. (e-h) Maximum filtered images. (i-l) Atomic models with atoms to be rotated highlighted in red and atoms to be sputtered colored in green. The scale bar in panel a is $1 \mathrm{~nm}$.

In Figure 7 we examine the origin of the dislocation observed in the top right corner of Figure 5. The dislocation was initially a part of the GB (colored in yellow in Figure 7e), before being detached from the loop by migrating northeastwards (Figure $7 \mathrm{c}, \mathrm{f}$ ). This motion is equivalent to a basic glide plus basic climb steps. After that it continued migrating as an isolated dislocation via both bond rotation and atom evaporation just as previously reported. ${ }^{12,28,30}$ The migration path over the next $12 \mathrm{~min}$ and $18 \mathrm{~s}$ is illustrated in Figure $7 \mathrm{~g}$. Our prior work has suggested that it is of equal probability for a dislocation to glide along both directions in the slip plane, if there is no other defect nearby for the dislocation to interact with. ${ }^{12}$ However, in Figure 7 it is apparent that the 
dislocation is inclined to glide away from GB loop, indicating that there may be a mutually exclusive interaction between them.

The process through which the GB loop in Figure 2a was observed to unwind is shown in Figure 8. The specimen temperature was maintained at $500^{\circ} \mathrm{C}$ in the beginning. The "tail" of the defect (Figure 8a) was first relaxed within 4 min 47 s. The GB loop was relatively stable in the next 3 min $33 \mathrm{~s}$ with only minor structural changes taking place in the meantime (Figure $8 \mathrm{~b}, \mathrm{c}$ ). Increasing the temperature to $800^{\circ} \mathrm{C}$ afterwards resulted in the accelerated shape evolution of the defect, shown in Figure $8 \mathrm{~g}-\mathrm{i}$. Figure $8 \mathrm{~g}$ is not as clearly resolved as other images since the structural change happened so quickly, leaving insufficient time to adjust the thermal-expansion induced focus change. But the shape of the GB loop can still be recognized from the contrast inversion between the pristine lattice and the rotated one inside the loop. Similar to what we observed at $500^{\circ} \mathrm{C}$, the defect separated into two smaller loops (Figure 8h), which reconfigured into a flower defect and a line defect respectively in Figure 8i. Both of them were observed to annealed back to pristine lattice in $8 \mathrm{~min} 11 \mathrm{~s}$ (Figure $8 \mathrm{~g}-\mathrm{i}$ and 9 ). It is also worth noticing that the Burger's vector for the defect was initially 0 and became $(1,0)$ after the disappearance of the "tail" and was back to 0 again after the splitting into two parts (Figure 81). One reasonable speculation is that the line defect attached to the GB loop in Figure 8a reconstructed into an isolated dislocation which soon after jumped out of the imaging area. Figure $8 \mathrm{c}$ and i there could be another dislocation emission event like in Figure 7, thus giving rise to the changes in the Burger's vector. 

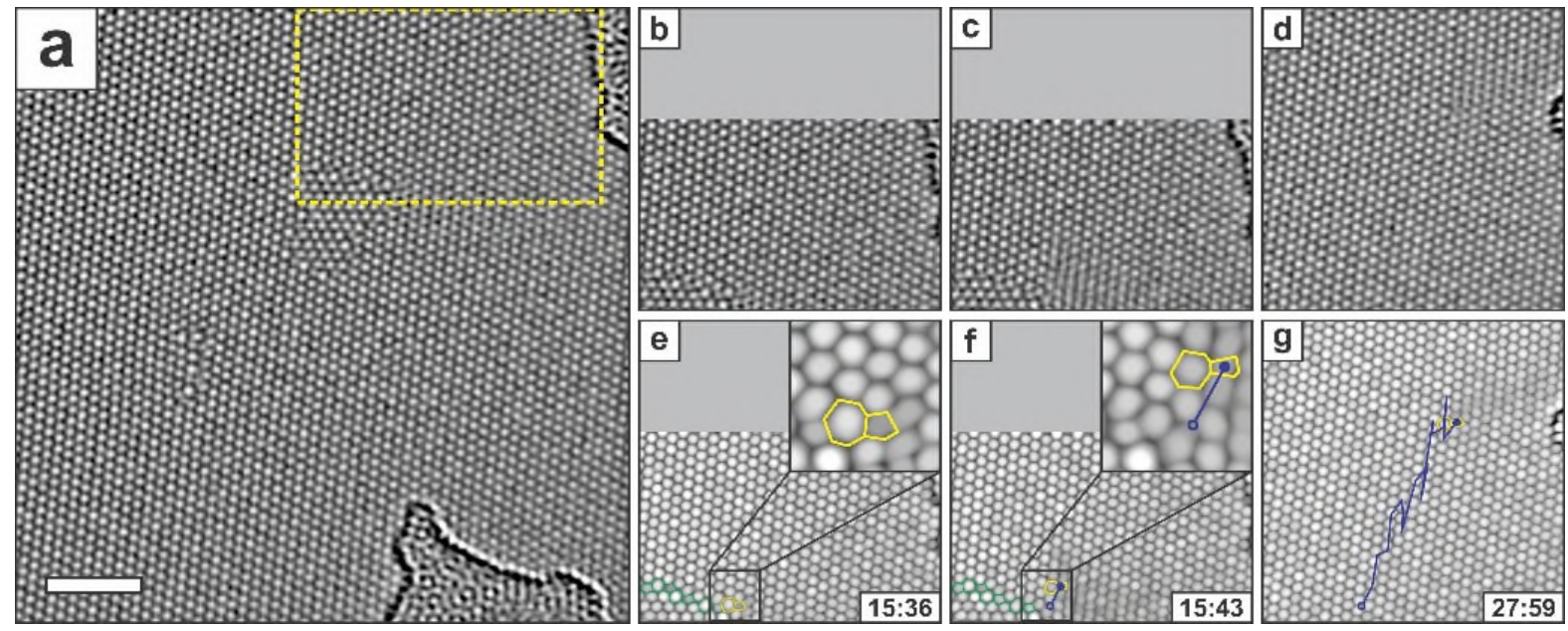

Figure 7. Ejection of a 5-7 dislocation during the unwinding process of the GB loop. (a) AC-TEM image showing the same GB loop as in Figure 4 at $15 \mathrm{~min} 36 \mathrm{~s}$ with the top right area cropped by the dashed box and presented in (b). (b-d) Time sequential images showing the separation of a 5-7 dislocation from the loop. (e-g) Maximum filtered images of $(b-d)$, with the magnified view of the dislocation shown in the inset. The trajectory of the dislocation over the whole time period is presented in $(\mathrm{g})$. The scale bar in panel a is $2 \mathrm{~nm}$.

In Figure 9, we study the structure and stability of the line defect cropped in Figure 8i by showing time series frames at higher magnification. We find that the line defect is actually the assembly of 5 adatom defects with the atomic structure we showed in Figure 1a. Prior work on adatom defects have been limited to smaller point defect structures with no observations of line defect made of carbon adatoms. This long line adatom defect is unexpected since the graphene lattice keeps losing atoms over the imaging period as a result of irradiation damage. The 5 added carbon dimers were observed to be sputtered one after another in Figure 9. Robertson et al. have demonstrated that adatom defects are energetically unfavorable under $80 \mathrm{kV}$ electron irradiation and the life time for high-order adatom defects (containing 4/6 additional atoms) is on a $\sim 30 \mathrm{~s}$ time scale at room temperature. ${ }^{20}$ However, in our experiment, the elimination of the line defect takes place in a deliberate manner at $800^{\circ} \mathrm{C}$ and the average life span of each single adatom defect is ca. $112 \mathrm{~s}$. If we further consider that the sputtering of carbon atom is far more likely to occur at $800{ }^{\circ} \mathrm{C}$ than at 
room temperature, ${ }^{12}$ we may conclude that the stability of adatom defect is enhanced by generating a string of them, which makes its application in graphene defect nanoengineering more promising.

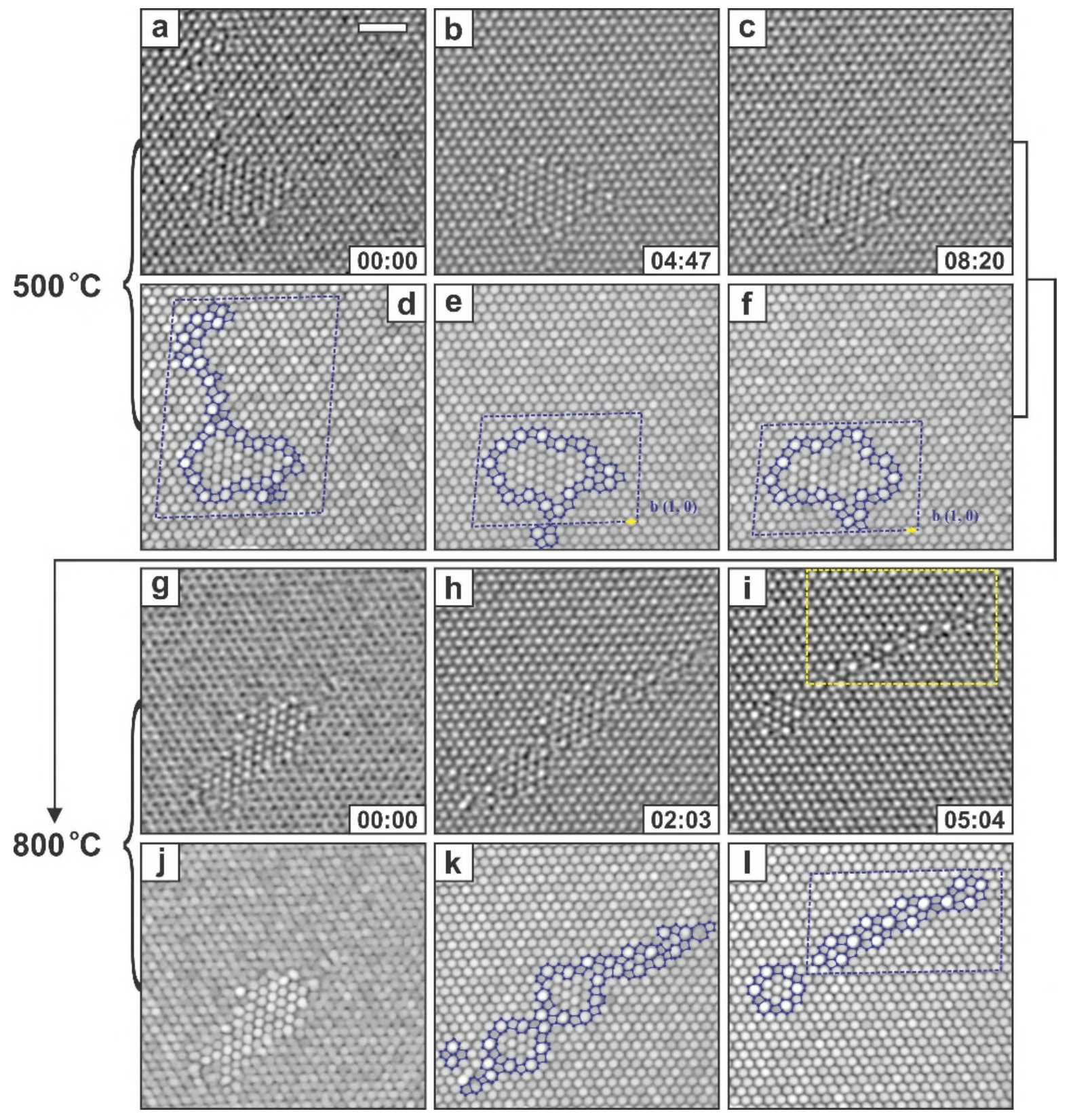

Figure 8. Time series of AC-TEM images showing the evolution of the GB loop from Figure 2a. (a-c, g-i) Gaussian filtered images. The temperature was increased from $500{ }^{\circ} \mathrm{C}$ to $800{ }^{\circ} \mathrm{C}$ between panel c and g. $(\mathrm{d}-\mathrm{f}, \mathrm{j}-\mathrm{k})$ Maximum filtered images with non-hexagonal rings, Burger's circuit and Burger's vector marked. The scale bar in panel a is $1 \mathrm{~nm}$. 

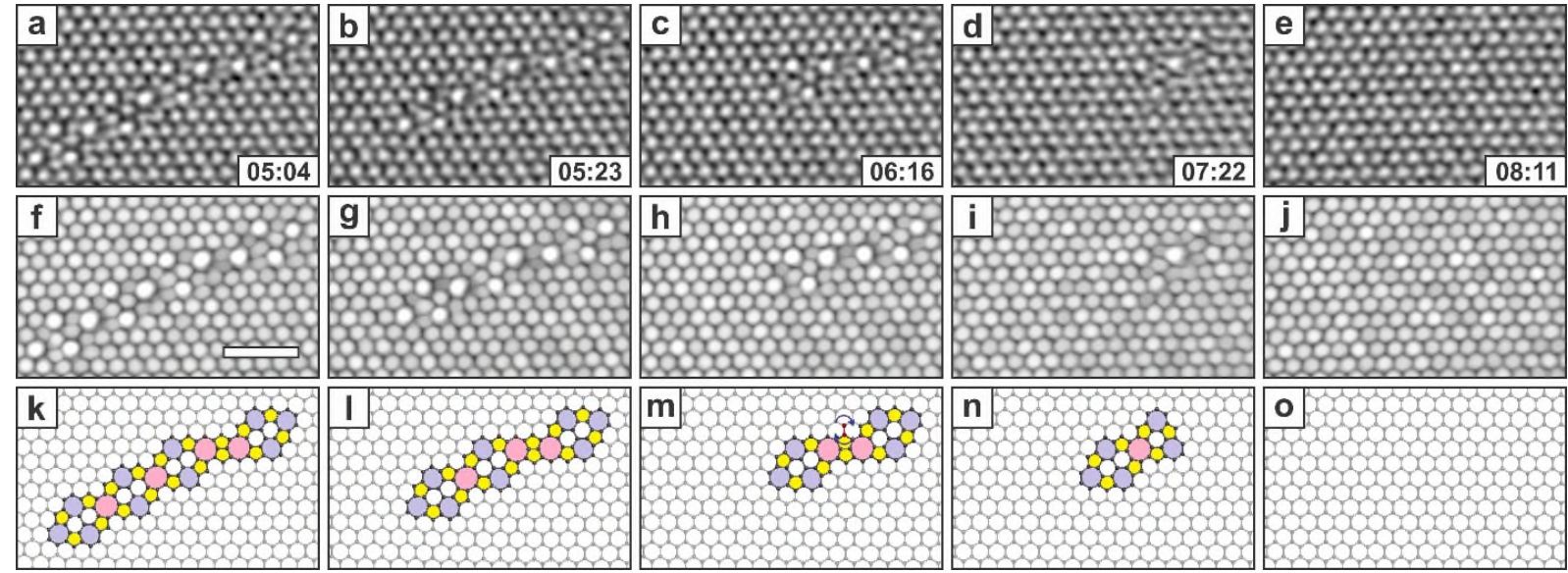

0

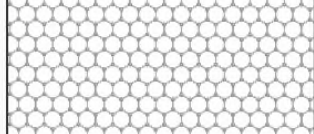

Figure 9. Evolution of a line defect assembled by carbon adatom defects. (a-e) Gaussian filtered AC-TEM images. Panel a shows magnified view of the cropped area in Figure 8i. (f-j) Maximum filtered images. (k-o) Corresponding atomic models. The scale bar in panel $\mathrm{f}$ is $1 \mathrm{~nm}$.

\section{Conclusion}

In summary, we have used high temperature in situ AC-TEM to investigate the formation, evolution and annihilation of large closed GB loop defects in graphene with atomic resolution. We have shown that the unwinding of GB loop is accompanied with splitting into smaller loops and the emission of 5-7 dislocations. The GB loop can never be fully relaxed under beam irradiation with the final state being isolated dislocations nanometers away from one another. During the evolution process we have also discovered the formation of a line defect consisting of several adjacent adatom defects, which is relatively robust even at $800{ }^{\circ} \mathrm{C}$. We also showed that the flower defect consisting of 6 bond rotations in graphene could be produced by the unwinding of more complicated defect clusters, indicating that it originates from a top down change of larger closed loop structures into smaller ones, rather than from the bottom up by direct bond rotations. We hope that this work can provide insights into the stability and dynamics of high order defects in graphene at elevated temperatures. 


\section{Methods}

\section{Synthesis of graphene}

Monolayer graphene was synthesized by atmospheric pressure chemical vapor deposition (CVD) method using a melted copper sheet as the catalytic as previously reported. ${ }^{19,31}$ The high purity copper foil (Alfa Aesar, Puratonic 99.999\% pure, $0.1 \mathrm{~mm}$ thick) of $\sim 1 \mathrm{~cm}^{2}$ were placed on the molybdenum piece of same size(Alfa Aesar, $99.95 \%$ pure, $0.1 \mathrm{~mm}$ thick), which were both loaded into a 1 inch quartz tube in the CVD system. Molybdenum act as a stable wetting layer to prevent liquid copper from balling. 100 s.c.c.m. $\mathrm{H}_{2} / \mathrm{Ar}\left(20 \% \mathrm{H}_{2}\right.$ in $\left.\mathrm{Ar}\right), 100$ s.c.c.m. $\mathrm{CH}_{4}\left(1 \% \mathrm{CH}_{4}\right.$ in $\left.\mathrm{Ar}\right)$ and 200 s.c.c.m. $100 \%$ Ar were flowed for 30 minutes. $\mathrm{CH}_{4}$ flow was switched off before increasing hot-zone temperature to $1090{ }^{\circ} \mathrm{C}$. Once the temperature reached $1090{ }^{\circ} \mathrm{C}$, the quartz tube was moved from the room temperature zone to the center of heating zone and annealed for 30 minutes. The flow of $\mathrm{H}_{2} / \mathrm{Ar}$ was then reduced to 80 s.c.c.m. and 10 s.c.c.m. of $1 \% \mathrm{CH}_{4} \mathrm{in} \mathrm{Ar}$ was added for 90 min for graphene growth. After growth, the quartz was removed from the heating zone for rapid cooling in the air with $\mathrm{CH}_{4}$ off.

\section{Transfer}

A PMMA scaffold ( $8 \% \mathrm{wt}$ in anisole, $495 \mathrm{k}$ molecular weight) was spin-coated onto the graphene sheet at $4700 \mathrm{rpm}$ for $60 \mathrm{~s}$ and then baked at $180{ }^{\circ} \mathrm{C}$ for $90 \mathrm{~s}$ to solidify. Afterwards the sample was made up of a molybdenum/copper/graphene/PMMA stack. The copper layer were etched by floating the sample on the mixed solution of iron (III) chloride and hydrochloride, leaving a floating graphene-PMMA film on the top after $48 \mathrm{~h}$. The film was collected using a clean glass slide and transferred onto the surface of the DI water for 30 min wash away remaining iron (III) chloride. To further dissolve excess iron chloride, the sample was transferred onto a $10 \%$ hydrogen 
chloride solution for $5 \mathrm{~min}$, before rinsed again in the DI water for $30 \mathrm{~min}$. The film was then transferred onto a SiN TEM grid designed for in-situ Transmission Electron Microscopy in a heating holder (DENSsolutions single tilt $30^{\circ}$ fitted with DENSsolutions High Temperature EM heater chip with a maximum operating temperature up to $800^{\circ} \mathrm{C}$ ). The thin $\mathrm{SiN}$ membrane on the heating holder contained several windows ( FIB prior to graphene transfer. These windows were essential to enable HRTEM imaging of the graphene lattice without contrast from the $\mathrm{SiN}$ membrane. The grid was then cured at $350{ }^{\circ} \mathrm{C}$ for $12 \mathrm{~h}$ to burn out PMMA, leaving clean graphene.

\section{Electron Microscopy}

AC-TEM images were taken at an accelerating voltage of $80 \mathrm{kV}$, using Oxford's JEOL JEM2200MCO field emission transmission electron microscope with a CEOS image corrector. ${ }^{28} \mathrm{~A}$ double Wien filter monochromator with a $7 \mu \mathrm{m}$ slit was used to reduce the energy spread of the electron beam to $\sim 0.21 \mathrm{eV}$. Data were recorded using a Gatan Ultrascan $4 \mathrm{~K} \times 4 \mathrm{~K}$ CCD camera with $2 \mathrm{~s}$ acquisition times and 2 pixel binning. TEM images were processed using Image J. Smoothing of images was achieved by using a Gaussian blur filter in Image J.

\section{In situ Heating Holder}

To perform variable-temperature experiments, we used a commercially available in situ heating holder from DENS Solutions (SH30-4M-FS). In the DENS Solutions holder, heating the sample was achieved by passing a current through a platinum resistive coil imbedded in the TEM chip (DENS Solutions DENS-C-30). The resistance of the platinum coil is monitored in a four-point configuration, and the temperature is calculated using the Callendar-Van Dusen equation (with calibration constants provided by the manufacturer). 


\section{Acknowledgment}

J.H.W. thanks the support from the Royal Society. C.G. thanks the support from the Clarendon Fund.

\section{References}

(1) Novoselov, K. S.; Geim, A. K.; Morozov, S. V; Jiang, D.; Zhang, Y.; Dubonos, S. V; Grigorieva, I. V; Firsov, A. A. Electric Field Effect in Atomically Thin Carbon Films. Science 2004, 306, 666-669.

(2) Castro Neto, a. H.; Peres, N. M. R.; Novoselov, K. S.; Geim, A. K. The Electronic Properties of Graphene. Rev. Mod. Phys. 2009, 81, 109-162.

(3) Lee, C.; Wei, X.; Kysar, J. W.; Hone, J. Measurement of the Elastic Properties and Intrinsic Strength of Monolayer Graphene. Science 2008, 321, 385-388.

(4) Boukhvalov, D. W.; Katsnelson, M. I. Chemical Functionalization of Graphene with Defects. Nano Lett. 2008, 8, 4374-4379.

(5) Zhang, Y.-H.; Chen, Y.-B.; Zhou, K.-G.; Liu, C.-H.; Zeng, J.; Zhang, H.-L.; Peng, Y. Improving Gas Sensing Properties of Graphene by Introducing Dopants and Defects: A FirstPrinciples Study. Nanotechnology 2009, 20, 185504.

(6) Albrecht, T. R.; Mizes, H. A.; Nogami, J.; Park, S.; Quate, C. F. Observation of Tilt Boundaries in Graphite by Scanning Tunneling Microscopy and Associated Multiple Tip Effects. Appl. Phys. Lett. 1988, 52, 362-364.

(7) Huang, P. Y.; Ruiz-Vargas, C. S.; van der Zande, A. M.; Whitney, W. S.; Levendorf, M. P.; Kevek, J. W.; Garg, S.; Alden, J. S.; Hustedt, C. J.; Zhu, Y.; et al. Grains and Grain Boundaries in Single-Layer Graphene Atomic Patchwork Quilts. Nature 2011, 469, 389-392.

(8) An, J.; Voelkl, E.; Suk, J. W.; Li, X.; Magnuson, C. W.; Fu, L.; Tiemeijer, P.; Bischoff, M.; Freitag, B.; Popova, E.; et al. Domain (Grain) Boundaries and Evidence of "Twinlike" Structures in Chemically Vapor Deposited Grown Graphene. ACS Nano 2011, 5, 2433-2439.

(9) Kurasch, S.; Kotakoski, J.; Lehtinen, O.; Skákalová, V.; Smet, J.; Krill, C. E.; Krasheninnikov, A. V; Kaiser, U. Atom-by-Atom Observation of Grain Boundary Migration in Graphene. Nano Lett. 2012, 12, 3168-3173.

(10) Yang, B.; Xu, H.; Lu, J.; Loh, K. P. Periodic Grain Boundaries Formed by Thermal Reconstruction of Polycrystalline Graphene Film. J. Am. Chem. Soc. 2014, 136, 12041-12046.

(11) Lahiri, J.; Lin, Y.; Bozkurt, P.; Oleynik, I. I.; Batzill, M. An Extended Defect in Graphene as a Metallic Wire. Nat. Nanotechnol. 2010, 5, 326-329.

(12) Gong, C.; Robertson, A. W.; He, K.; Lee, G.-D.; Yoon, E.; Allen, C. S.; Kirkland, A. I.; Warner, J. H. Thermally Induced Dynamics of Dislocations in Graphene at Atomic Resolution. ACS Nano 2015, 9, 10066-10075. 
(13) Westenfelder, B.; Meyer, J. C.; Biskupek, J.; Kurasch, S.; Scholz, F.; Krill, C. E.; Kaiser, U. Transformations of Carbon Adsorbates on Graphene Substrates under Extreme Heat. Nano Lett. 2011, 11, 5123-5127.

(14) Grantab, R.; Shenoy, V. B.; Ruoff, R. S. Anomalous Strength Characteristics of Tilt Grain Boundaries in Graphene. Science 2010, 330, 946-948.

(15) Wei, Y.; Wu, J.; Yin, H.; Shi, X.; Yang, R.; Dresselhaus, M. The Nature of Strength Enhancement and Weakening by Pentagon-heptagon Defects in Graphene. Nat. Mater. 2012, 11, 759-763.

(16) López-Sancho, M. P.; de Juan, F.; Vozmediano, M. A. H. Magnetic Moments in the Presence of Topological Defects in Graphene. Phys. Rev. B 2009, 79, 75413.

(17) Yasaei, P.; Kumar, B.; Hantehzadeh, R.; Kayyalha, M.; Baskin, A.; Repnin, N.; Wang, C.; Klie, R. F.; Chen, Y. P.; Král, P.; et al. Chemical Sensing with Switchable Transport Channels in Graphene Grain Boundaries. Nat. Commun. 2014, 5, 4911.

(18) Khosravian, N.; Samani, M. K.; Loh, G. C.; Chen, G. C. K.; Baillargeat, D.; Tay, B. K. Effects of a Grain Boundary Loop on the Thermal Conductivity of Graphene: A Molecular Dynamics Study. Comput. Mater. Sci. 2013, 79, 132-135.

(19) Fan, Y.; He, K.; Tan, H.; Speller, S.; Warner, J. H. Crack-Free Growth and Transfer of Continuous Monolayer Graphene Grown on Melted Copper. Chem. Mater. 2014, 26, 4984-4991.

(20) Robertson, A. W.; He, K.; Kirkland, A. I.; Warner, J. H. Inflating Graphene with Atomic Scale Blisters. Nano Lett. 2014, 14, 908-914.

(21) Lusk, M. T.; Wu, D. T.; Carr, L. D. Graphene Nanoengineering and the Inverse StoneThrower-Wales Defect. Phys. Rev. B 2010, 81, 155444.

(22) Robertson, A. W.; Allen, C. S.; Wu, Y. A.; He, K.; Olivier, J.; Neethling, J.; Kirkland, A. I.; Warner, J. H. Spatial Control of Defect Creation in Graphene at the Nanoscale. Nat. Commun. 2012, 3, 1144.

(23) Li, L.; Reich, S.; Robertson, J. Defect Energies of Graphite: Density-Functional Calculations. Phys. Rev. B 2005, 72, 184109.

(24) Kim, Y.; Ihm, J.; Yoon, E.; Lee, G.-D. Dynamics and Stability of Divacancy Defects in Graphene. Phys. Rev. B 2011, 84, 075445.

(25) Robertson, A. W.; Lee, G.-D.; He, K.; Fan, Y.; Allen, C. S.; Lee, S.; Kim, H.; Yoon, E.; Zheng, H.; Kirkland, A. I.; et al. Partial Dislocations in Graphene and Their Atomic Level Migration Dynamics. Nano Lett. 2015, 15, 5950-5955.

(26) Jeong, B.; Ihm, J.; Lee, G.-D. Stability of Dislocation Defect with Two Pentagon-Heptagon Pairs in Graphene. Phys. Rev. B 2008, 78, 165403.

(27) Lee, G.-D.; Yoon, E.; He, K.; Robertson, A. W.; Warner, J. H. Detailed Formation Processes of Stable Dislocations in Graphene. Nanoscale 2014, 6, 14836-14844.

(28) Warner, J. H.; Margine, E. R.; Mukai, M.; Robertson, A. W.; Giustino, F.; Kirkland, A. I. Dislocation-Driven Deformations in Graphene. Science 2012, 337, 209-212. 
(29) Gong, C.; He, K.; Robertson, A. W.; Yoon, E.; Lee, G.-D.; Warner, J. H. Spatially Dependent Lattice Deformations for Dislocations at the Edges of Graphene. ACS Nano 2015, 9, 656-662.

(30) Lehtinen, O.; Kurasch, S.; Krasheninnikov, A. V.; Kaiser, U. Atomic Scale Study of the Life Cycle of a Dislocation in Graphene from Birth to Annihilation. Nat. Commun. 2013, 4, 2098.

(31) Wu, Y. A.; Fan, Y.; Speller, S.; Creeth, G. L.; Sadowski, J. T.; He, K.; Robertson, A. W.; Allen, C. S.; Warner, J. H. Large Single Crystals of Graphene on Melted Copper Using Chemical Vapor Deposition. ACS Nano 2012, 6, 5010-5017.

TOC Graphic

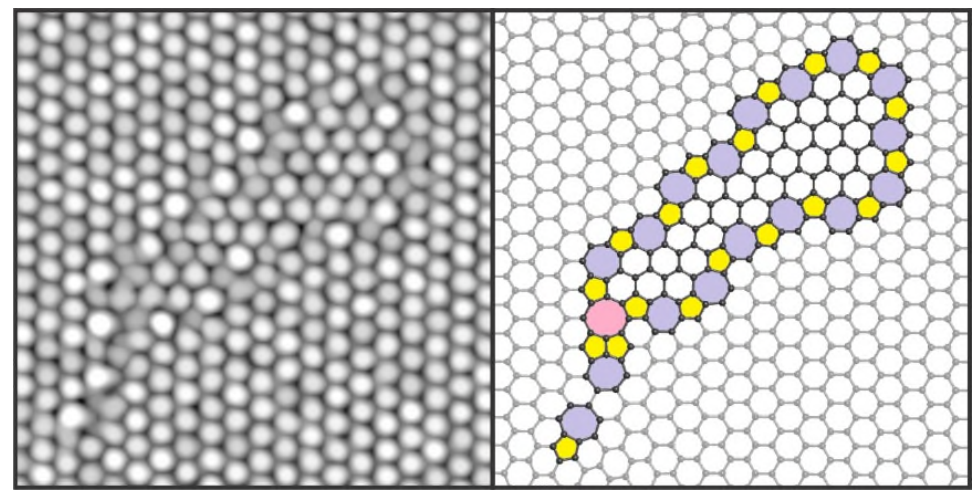

